This is the submitted version of the following article:

Li Z., Aranda-Ramos A., Güell-Grau P., Tajada J.L., Pou-Macayo L., Lope Piedrafita S., Pi F., G. Roca A., Baró M.D., Sort J., Nogués C., Nogués J., Sepúlveda B.. Magnetically amplified photothermal therapies and multimodal imaging with magneto-plasmonic nanodomes. Applied Materials Today, (2018). 12. : 430 - . 10.1016/j.apmt.2018.07.008,

which has been published in final form at https://dx.doi.org/10.1016/j.apmt.2018.07.008 @ https://dx.doi.org/10.1016/j.apmt.2018.07.008. This manuscript version is made available under the CC-BY-NC-ND 4.0 license http://creativecommons. org/licenses/by-nc-nd/4.0/ 


\section{Magnetically amplified photothermal therapies and multimodal imaging with magneto- plasmonic nanodomes}

Zhi Li, ${ }^{a, d}$ Antonio Aranda-Ramos, ${ }^{b}$ Pau Güell-Grau, ${ }^{a}$ José Luis Tajada, ${ }^{a}$ Laia Pou-Macayo, ${ }^{b}$ Silvia Lope Piedrafita, ${ }^{c}$ Francesc Pi, ${ }^{d}$ Alejandro Gómez Roca, ${ }^{a}$ María Dolors Baró, ${ }^{d}$ Jordi Sort, ${ }^{\text {,e }}$ Carme Nogués, ${ }^{b}$ Josep Nogués, ${ }^{a, e}$ and Borja Sepúlveda ${ }^{a, *}$

${ }^{a}$ Catalan Institute of Nanoscience and Nanotechnology (ICN2), CSIC and BIST, Campus UAB, Bellaterra, 08193 Barcelona, Spain

${ }^{b}$ Departament de Biologia Cel-lular, Fisiologia i Immunologia, Facultat de Biociències, Universitat Autònoma de Barcelona, Bellaterra, 08193 Barcelona, Spain.

${ }^{c}$ Servei de Ressonància Magnètica Nuclear, Universitat Autònoma de Barcelona, Bellaterra, 08193 Barcelona, Spain, and Centro de Investigación Biomédica en Red en Bioingeniería, Biomateriales y Nanomedicina (CIBER-BBN).

${ }^{d}$ Departament de Física, Facultat de Ciències, Universitat Autònoma de Barcelona, Bellaterra, 08193 Barcelona, Spain.

e ICREA, Pg. Lluís Companys 23, 08010 Barcelona, Spain.

Keywords: Magneto-plasmonic nanoparticles, Photothermal therapies, Magnetic manipulation, Contrast agents.

\section{ABSTRACT}

Nanotherapies require new ways for controlling and improving the delivery of the therapeutic agents to the site of action to maximize their efficacy and minimize the side effects. This control is particularly relevant in photothermal treatments to reduce the required light intensity and amount of injected nanoparticles, and to minimize necrotic cell deaths. Here we present a novel concept for multifunctional nanobiomedical agents: magneto-plasmonic (MP) nanodomes for magnetically guided and amplified photothermal therapies and as contrast agents for multimodal imaging. The MP nanodomes are composed of a Fe/Au bilayer semi-shell deposited on a $100 \mathrm{~nm}$ diameter fluorescent polystyrene nanosphere, which gather a unique combination of straightforward functionalization, high colloidal stability, very strong ferromagnetic behavior and intense optical absorption efficiency in the near infrared. We show that the photothermal 
conversion efficiency of the Fe/Au nanodomes with high Fe ratios is substantially larger than pure plasmonic Au nanodomes and the state-of-art plasmonic nanoheaters, i.e. Au nanorods and nanoshells, by merging strong optical absorption, minimized scattering and low optical anisotropy. Remarkably, the effective magnetophoretic concentration of the Fe/Au nanodomes at the illumination region enables large local increase of the optically induced temperature rise. The Fe semishell also provides very intense $T_{2}$ contrast in nuclear magnetic resonance, which is at least 15-fold larger per particle than commercial iron oxide contrast agents. Moreover, the fluorescent polystyrene nanosphere and the Au semishell integrate valuable fluorescent and Xray contrasts, respectively, which we have used to assess the nanodomes internalization by cancer cells. The MP nanodomes are nontoxic to cells even in the case of magnetophoretic local enrichment with initially high particle concentration $(100 \mu \mathrm{g} / \mathrm{mL})$. Remarkably, we demonstrate amplified local photothermal treatments by the magnetic enrichment of the nanodomes at the illumination region, which enables reaching nearly $100 \%$ reduction of cell viability with low particle concentration $(10 \mu \mathrm{g} / \mathrm{mL})$ and mild NIR laser intensity $\left(5 \mathrm{~W} / \mathrm{cm}^{2}\right)$. These results highlight the high potential of MP nanodomes for magnetically guided and amplified photothermal therapies. 


\section{Introduction}

Nanotherapies are playing an increasingly important role to create new therapies with higher efficacy and lower side effects than traditional chemical treatments, especially in diseases such as cancer [1-4]. In addition to efficient drug carriers, nanoparticles can also be the source of efficient physical therapies, such as local hyperthermia, which can be employed to thermally destroy the tumors [5-9] or as adjuvant of chemotherapies [10-14]. Photothermal therapies are typically based on plasmonic nanoparticles due to the amplified optical absorption associated with their localized surface plasmon resonance (LSPR) $[15,16]$, which can be tuned to the nearinfrared (NIR), where the skin and capillaries show higher transparency [17]. However, the passive delivery of nanoparticles hampers their control and efficient concentration at the tumor. The main obstacles that passively delivered nanoparticles encounter in cancer therapies are [18]: i) hepatic, renal or immune system clearance, which can drastically reduce the probability to target the tumor; ii) less pronounced enhanced permeability and retention effect in clinical tumors than in murine models, iii) inherent elevated interstitial hydrostatic pressure in solid tumors that inhibits nanoparticle extravasation, and iv) poor diffusion inside solid tumors due to abnormally high cross-linked extracellular matrix. An attractive way to overcome these hurdles is the external control and guidance of the nanoparticles to the site of action. The ability of magnetic nanoparticles to respond to magnetic forces has fueled the development of nanostructures to magnetically propel the nanoparticles with external magnetic fields [19-24]. Therefore, the combination of plasmonic and magnetic materials can be an interesting alternative to overcome the drawbacks associated to the passive delivery of the nanoparticles. Magnetoplasmonic nanoparticles, in addition to enhanced magneto-optic effects [25,26], could combine efficient plasmonic light absorption with magnetic manipulation [27-30]. 
To date, the majority of colloidal magneto-plasmonic nanostructures are based on merging plasmonic nanoparticles with small superparamagnetic iron oxide nanoparticles (SPIONs) [3033]. However, the small size and weak magnetic moment of the SPIONs, required to minimize their magnetic interaction (which is crucial to ensure the colloidal stability), drastically limits their magnetic actuation capabilities.

Moreover, colloidal magnetic, plasmonic and magneto-plasmonic nanoparticles are generally obtained in organic solvents by chemical synthesis, in which achieving controlled optical and magnetic properties with narrow size and shape distributions can be complex. In addition, the biomedical applications require transferring the nanostructures to an aqueous medium and their functionalization, which are cumbersome processes in nanoparticles obtained from organic-based syntheses that can lead to particle aggregation, especially when heterostructures are involved. In contrast, scalable top-down methods capable of generating macroscopic amounts of colloidal nanostructures in a simple and effective way are considerably less developed. Therefore, achieving cost-effective ways to fabricate multifunctional nanostructures with highly controlled and strong optical and magnetic properties in a large scale may lead to new high-added value biomedical agents.

Here we present novel colloidal ferromagnetic/plasmonic $\mathrm{Fe} / \mathrm{Au}$ nanodomes enabling: i) excellent colloidal stability; ii) high optical heating efficiency in the NIR comparable to that of state-of-art plasmonic nanoparticles; iii) strong magnetic manipulation via magnetophoretic forces, and iv) very high contrast for fluorescence, nuclear magnetic resonance (NMR) and Xray imaging. We show that the combination of magnetophoretic manipulation and optical heating within the magneto-plasmonic (MP) nanodomes enables a near 100\% reduction of cell viability 
in photothermal treatments in vitro under demanding conditions of low MP nanodomes concentration $(10 \mu \mathrm{g} / \mathrm{mL})$ and mild NIR laser intensity $\left(5 \mathrm{~W} / \mathrm{cm}^{2}\right)$.

\section{Experimental}

\subsection{Fabrication of the MP nanodomes}

Unlike typical chemical synthesis methods, the MP nanodomes are fabricated by a combination of colloidal nanolithography [34] and physical vapor deposition, which are scalable and cost-effective processes that enable accurate control of their magnetic and optical properties. A schematic drawing of the different fabrication steps can be seen in Fig. S1 in the Supporting Information section. Briefly, we employed four-inch silicon wafers (Siegert Wafer $\mathrm{GmbH}$ ) cleaned with oxygen plasma (PS210, PVA Tepla America, Inc.) as substrates for the electrostatic self-assembly of the polystyrene nanospheres. The self-assembly starts by incubating the wafer surface with a solution containing positively charged polyelectrolyte [poly(diallyldimethylammonium chloride, PDDA, Sigma-Aldrich] at $2 \%$ concentration for 1 min. Then the wafer is rinsed with osmotized water and blow dried with $\mathrm{N}_{2}$ gas, thereby yielding a monolayer of PDDA on the surface. Next, the modified wafer surface is incubated with the dispersion of sulfate functionalized polystyrene beads $(100 \mathrm{~nm}$ diameter, concentration $0.2 \%$, Life Technologies) for $2 \mathrm{~min}$, followed by rinsing with water and drying with $\mathrm{N}_{2}$ flow to obtain the self-assembled monolayer of nanospheres. The exposed surface of nanospheres on the wafer was then coated with $\mathrm{Fe}$ and Au layers using electron beam evaporator (UNIVEX 450, Leybold $^{\circledR}$ ) to form the Fe/Au bilayer semi-shells with a total thickness of $40 \mathrm{~nm}$. The thickness of the deposited bilayers was monitored with Ångstrom precision by a quartz crystal microbalance during the evaporations. To improve the stability in aqueous solutions, the MP 
nanodomes were directly functionalized, while still on the wafer, with the negative charged polyelectrolyte [Poly (sodium 4-styrenesulfonate, PSS, Sigma-Aldrich] by incubating patterned surface in at $2 \%$ concentration water solution for $3 \mathrm{~min}$ to deposit a monolayer on the $\mathrm{Au}$ surface. The wafer is rinsed with water to remove the excess of PSS and is dried with $\mathrm{N}_{2}$ flow. The surface functionalization with proteins (e.g. for cell targeting) can also be carried out directly on the nanopatterned substrate without the need of any chemical linker. In this case we exploit the very high affinity of amine and cysteine groups of the proteins towards bare $\mathrm{Au}$ layers. We have actually observed the formation of uniform and stable protein monolayers on the $\mathrm{Fe} / \mathrm{Au}$ nanodomes by incubating the nanopatterned substrate with a solution of $10 \mu \mathrm{g} / \mathrm{mL}$ of protein in water for just $1 \mathrm{~h}$ (Fig. S2).

To disperse the particles in water, the wafer together with $10 \mathrm{~mL}$ of water was introduced into an ultrasonic bath for $1 \mathrm{~min}$. The MP nanodomes were finally concentrated and redispersed through centrifugation (4000 rpm, $5 \mathrm{~min}$ ), followed by ultrasonication to achieve highly stable dispersions at the required concentrations. The Si wafers can be reused after cleaning for $10 \mathrm{~min}$ in aqua regia, which efficiently dissolves the Fe and Au layers.

\subsection{Morphological, optical, magnetic and colloidal characterization}

To study the size and distribution of MP nanodomes on the Si wafers, scanning electron microscopy (SEM) studies were performed using Quanta SEM 650 (Field Electron and Ion Company (FEI)) at $20 \mathrm{kV}$. The density of nanospheres coated on the wafer was quantified by using ImageJ software. Transmission electron microscopy, TEM, images and electron energy loss spectroscopy (EELS) analysis were performed in a FEI Tecnai F20 equipped with a Quantum GIF EELS spectrometer. 
The stability of water dispersed MP nanodomes was studied through dynamic light scattering using Zetasizer Nano ZS (Malvern Instruments Ltd.). Both Zeta potential and average size of particles were simultaneously obtained. The visible-near infrared (vis-NIR) spectroscopy studies of the MP nanodomes dispersions were carried out using Lambda25 (PerkinElmer). For the optical measurements, the optical spectra ranged from $400 \mathrm{~nm}$ to $1100 \mathrm{~nm}$, which covered visible light and near infrared region. The concentration of the MP dispersions was determined by ICPOES (Perking Elmer Optima 4300DV).

The magnetic characterization of the MP nanodomes was performed on monolayers that were transferred to adhesive tapes to eliminate the magnetic signal from the bilayer that is deposited on the wafer surface. Magnetization loops were acquired at room temperature using a vibrating sample magnetometer (MicroSense, LOT QuantumDesign) with a maximum applied field of 20 kOe. The measurements were performed by applying the field either parallel or perpendicular to the sample, i.e., in-plane or out-of-plane conditions.

\subsection{Photothermal characterization}

A custom-made photothermal testing system was used to determine the photothermal conversion efficiency of MP nanodomes in water (see Fig. S3), which consists of: i) a NIR laser diode with emission wavelength at $808 \mathrm{~nm}$ (L808P500MM, Thorlabs) driven by a laser diode controller (LDC240C, Thorlabs) and a temperature controller (TED240C, THORLABS), ii) an optical collimating and aligning system, iii) an infrared thermometer (MLX90614, Melexis) to monitor the temperature variations at the liquid surface, iv) a power meter (PM100D, Thorlabs), and v) a computer with the data acquisition software. The laser incident power upon the samples was $166 \mathrm{~mW}$. For the analysis, samples with different concentration and constant volume (400 
$\mu \mathrm{L})$ were prepared. The temperature of the solution and the power transmitted through the sample were continuously monitored during the assays. The time interval of data recording for both transmitted power and instant temperature was 1s. The experimental photothermal conversion efficiency comparison with $\mathrm{Au}$ nanorods and nanoshells was carried out with Nanocomposix commercial particles, with references GRCN800 and GSPN800, respectively.

To analyze the magnetic manipulation of MP nanodomes by magnetophoretic forces, a stack of cylindrical FeNdB magnets (6 mm diameter with a 2500 Oe field at the surface) were attached at the lateral side of the cuvettes.

\subsection{Nuclear Magnetic Resonance}

${ }^{1} \mathrm{H}$-magnetic resonance imaging (MRI) studies were performed in a $70 \mathrm{kOe}$ Bruker BioSpec 70/30 USR (Bruker BioSpin GmbH, Ettlingen, Germany) system equipped with a mini-imaging gradient set $(4000 \mathrm{Oe} / \mathrm{m})$ and using a linear volume coil with $72 \mathrm{~mm}$ inner diameter. Magnetic resonance data were acquired and processed on a Linux computer using Paravision 5.1 software (Bruker BioSpin GmbH, Ettlingen, Germany). For the relaxitivity measurements, phantoms containing MP nanoparticles at various concentrations in 2\% agarose were prepared. Magnetic resonance images were obtained from two $2.5 \mathrm{~mm}$ slice thickness coronal sections with a field of view (FOV) of $9 \times 6 \mathrm{~cm}^{2}$. Longitudinal relaxation times $\left(\mathrm{T}_{1}\right)$ were measured using a spin echo sequence with variable repetition time $(\mathrm{TR}=300,500,700,1000,1300,1700,2000,2600,3500$, and $5000 \mathrm{~ms})$, echo time $=7.5 \mathrm{~ms}$, and matrix size $(\mathrm{MTX})=128 \times 128$. For transverse relaxation time $\left(\mathrm{T}_{2}\right)$ measurements, a multi-slice multi-echo sequence was used, with $\mathrm{TR}=3 \mathrm{~s}$, TE values between 10 and $600 \mathrm{~ms}$ in steps of $10 \mathrm{~ms}$, and $\mathrm{MTX}=128 \times 128$. Data were fitted to exponential curves to obtain the $T_{1}$ and $T_{2}$ relaxation times, respectively. Longitudinal and transverse 
relaxivity values, $r_{1}$ and $r_{2}$, were obtained as the slope of the linear regression of the relaxation rates $(R)$, as the inverse of the relaxation times $\left(R_{i}=1 / T_{i}, i=1,2\right)$ versus Fe concentration.

\subsection{Cell Culture}

Human Epithelial Cervical Adenocarcinoma Cells (HeLa, ATCC) were cultured in Minimal Essential Medium (Gibco, Life Technologies) supplemented with 10\% Fetal Bovine Serum (Gibco) and $2 \mathrm{mM} \mathrm{L-Glutamine} \mathrm{(Life} \mathrm{Technologies).} \mathrm{Cultures} \mathrm{were} \mathrm{maintained} \mathrm{at} 37{ }^{\circ} \mathrm{C}$ in a $5 \%$ $\mathrm{CO}_{2}$ humidified atmosphere (standard culture conditions).

2.6. Scanning electron microscopy of the cell cultures

Cells were seeded in glass bottom dishes (MatTek Corporation) at a density of $3 \times 10^{4}$ cells $/ \mathrm{mL}$ and incubated in standard conditions for $24 \mathrm{~h}$. Two concentrations of MP nanodomes were tested: 10 and $100 \mu \mathrm{g} / \mathrm{mL}$. MP nanodomes were added directly to the cell cultures and incubated in standard conditions for $3 \mathrm{~h}$ with or without the FeNdB spherical magnet underneath. To remove the non-internalized MP nanodomes, the cell media were replaced with fresh media and the cells were further treated with the laser according to each condition. After the MP nanodomes incubation and photothermal treatment, the cells were rinsed twice in $0.1 \mathrm{M}$ Sodium Cacodylate Buffer at pH 7.3 (SCB; TAAB), fixed in $2.5 \%$ Microscopy Glutaraldehyde solution for electron microscopy (Merck) in SCB for $25 \mathrm{~min}$ at room temperature and rinsed again twice in SCB. Cell dehydration was done in a series of ethanol washes (50, 70, 90 and twice $100 \%), 7$ min each. Finally, cells were dried in hexamethyldisilazane (HMDS; Electron Microscope Science) for 15 min after which HMDS was withdrawn and samples were let to dry overnight. Samples were mounted on special stubs and observed using a SEM (Zeiss Merlin). 


\subsection{Confocal Laser Scanning Microscopy (CLSM)}

Cells were seeded in glass bottom dishes (MatTek Corporation) at a density of $3 \times 10^{4}$ cells $/ \mathrm{mL}$ and incubated in standard conditions for $24 \mathrm{~h}$. Then, $10 \mu \mathrm{g} / \mathrm{mL}$ of fluorescent MP nanodomes $\left(\lambda_{\text {excitation }}=575, \lambda_{\text {emission }}=610 \mathrm{~nm}\right)$ were added directly to cell cultures and incubated for $3 \mathrm{~h}$ in standard conditions. After incubation, the cells were washed twice with saline solution and $1 \mathrm{~mL}$ of fresh medium was added to the sample. Finally, the cells were stained with $1 \mu \mathrm{l}$ of Cell ${ }^{\mathrm{TM}}$ Deep Red plasma membrane stain (Life Technologies), to reveal the plasma membrane, and 0.5

$\mu 1$ of Hoechst 33342 (Life Technologies) to expose the nucleus. Images were captured sequentially using an Olympus CLSM (Olympus FV1000). Three different lasers were used: 405, 559 and $635 \mathrm{~nm}$, to visualize nucleus, MP nanodomes and plasma membrane, respectively. Stacks of images along the z-axis were obtained for a selected area using the xyz mode of the CLSM and the ImageJ (Fiji) and Bio-formats plugins were used to obtain overlapped images of all channels (nuclei, plasma membranes and MP nanodomes) and the 3D reconstructions and cross-section projections were used to confirm MP nanodomes' internalization.

\subsection{Soft Transmission X-Ray microscopy}

Cells were seeded onto gold grids covered with FORMVAR and carbon foil at a density of $5 \times 10^{4}$ cells $/ \mathrm{mL}$ and incubated in standard conditions for $24 \mathrm{~h}$. Then, cell cultures were incubated in the presence or absence (control cultures) of $25 \mu \mathrm{g} / \mathrm{mL}$ of $\mathrm{MP}$ nanodomes for $3 \mathrm{~h}$ and afterwards, the incubation solution was withdrawn. The grids were subsequently rinsed in saline solution, plunge-freeze in liquid ethane chilled with liquid nitrogen and transported to the ALBA synchrotron. Finally, full field X-ray tomography was carried out at $520 \mathrm{eV}$ excitation energy at 
the Mistral beamline of the ALBA synchrotron light source to obtain carbon compounds within the cryopreserved cells in the so-called "water-window".

2.9. Photothermal treatment and cell death evaluation

Cells were seeded in cover glass-bottom dishes (MatTek, growth area $\sim 154 \mathrm{~mm}^{2}$ ) at a density of $6 \times 10^{4}$ cells $/ \mathrm{mL}$ and kept in standard culture conditions in $500 \mu \mathrm{l}$ of cell medium for $24 \mathrm{~h}$. Next, the cell cultures were incubated in the presence or absence (control cultures) of 10 or 100 $\mu \mathrm{g} / \mathrm{mL}$ of MP nanodomes for $3 \mathrm{~h}$. Then, the cells were washed three times with Hank's Balanced Salt Solution to remove the dispersed nanodomes in the cell medium. The cells were finally kept in $1 \mathrm{ml}$ of medium during the laser treatment and the overnight incubation after the treatment. The photothermal treatment was performed inside the incubator (HeraCell) using the light from a collimated fiber-coupled laser diode with emission wavelength at $808 \mathrm{~nm}$ (B1-808-1500-15A, Laser Components). The incident intensity in the sample was approximately $5 \mathrm{~W} / \mathrm{cm}^{2}$ and the treated area was a circle of $6 \mathrm{~mm}$ in diameter. The treatment was carried out for $30 \mathrm{~min}$. To evaluate the cytotoxicity of the photothermal treatment, after irradiation, cells were incubated again in standard conditions for $24 \mathrm{~h}$, and then incubated with the LIVE/DEAD ${ }^{\circledR}$ Viability and Cyototoxicity assay (Life Technologies) following manufacturer's guidelines. Images were acquired using the inverted fluorescence microscope Olympus IX71 (Olympus) and processed through ImageJ (Fiji). Three independent experiments were performed. The MP nanodomes photothermal effect was analyzed using Fisher's exact tests, using Graphpad Prism ${ }^{\circledR}$ 7.0a software (Graphpad Software). Statistical significance was considered when $\mathrm{P}<0.05$. 


\section{Results and discussion}

The MP nanodomes are composed of a $100 \mathrm{~nm}$ diameter polystyrene core that is partially coated by a Fe/Au bilayer semi-shell. To show their tunable magnetic and optical properties, in this study we vary the relative thickness of the Fe and Au layers, but we keep a total bilayer thickness of $40 \mathrm{~nm}$ (Fig. 1a). The fabrication process yields a monolayer of well-separated nanodomes with a short-range order distribution and homogeneous density of $1.6 \cdot 10^{9}$ nanodomes $/ \mathrm{cm}^{2}$ (Fig. 1b), which is equivalent to $c a \cdot 1.3 \cdot 10^{11}$ particles per wafer.

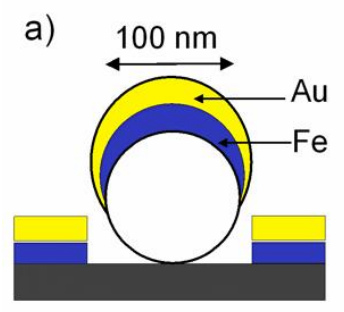

b)
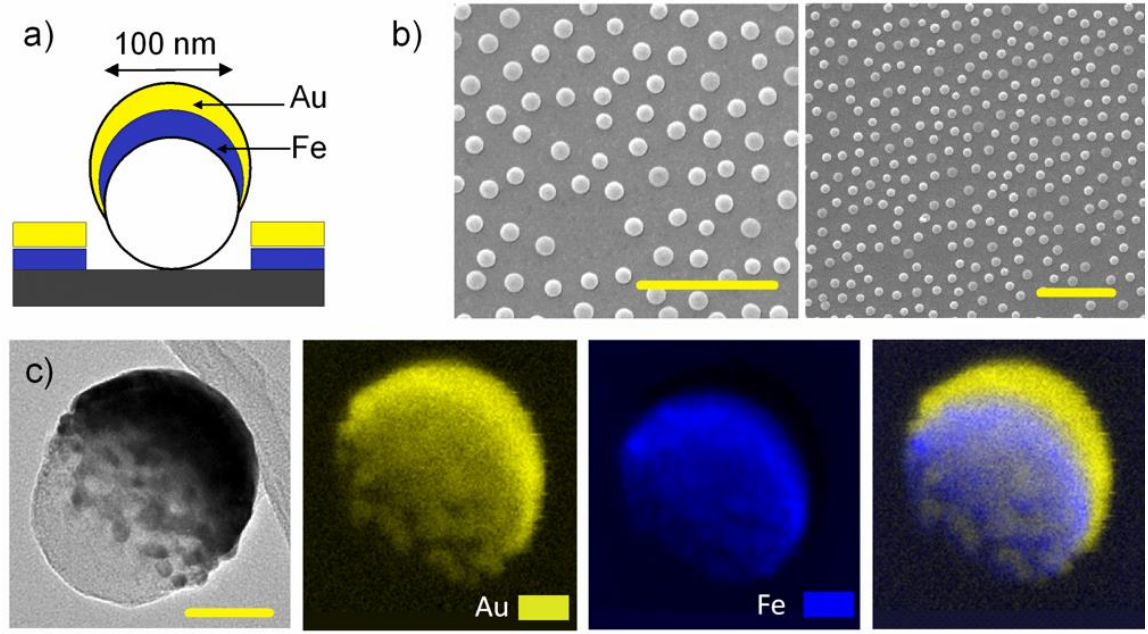

Fig. 1. Morphological and composition characterization of the MP nanodomes. a)

Schematic of the MP nanodomes composed of Fe and Au bilayers. b) SEM image of the self-assembled nanodomes on the wafer with a density of $1.6 \cdot 10^{9}$ particles $/ \mathrm{cm}^{2}$. The scale bars are $1 \mu \mathrm{m} . \mathrm{c})$ High angular annular dark field scanning transmission electron microscopy images of a MP nanodome with $20 \mathrm{~nm} \mathrm{Fe}$ and $20 \mathrm{~nm}$ Au together with the EELS mappings for the Au M-edge (yellow) and the Fe L-edge (blue) signal and the composed image. Scale bar $50 \mathrm{~nm}$. 
Mass spectrometry analyses of the MP nanodomes dispersions from different wafers concentrated in $1 \mathrm{~mL}$ of water have shown an average concentration of $(1.35 \pm 0.04) \cdot 10^{11}$ nanodomes/mL (see Supporting Information Table S1). Considering the estimated amount of particles per wafer obtained by the SEM analysis, virtually $100 \%$ of the self-assembled nanodomes on the wafer are transferred to the liquid. Dynamic light scattering measurements have shown an averaged hydrodynamic diameter of $114 \mathrm{~nm}$ (polydispersity index - PDI $=0.09$ ) and zeta-potential of $-25 \mathrm{mV}$. After more than six months storage at room temperature the nanodomes show an averaged diameter of $116 \mathrm{~nm}$ and similar PDI value, thereby demonstrating their extremely high colloidal stability even for ferromagnetic nanodomes with high Fe content.

The optical properties of the MP nanodomes in water for different Fe/Au ratios are gathered in Fig. 2a. The extinction spectrum of $40 \mathrm{~nm}$ thick Au nanodomes shows two peaks located at $650 \mathrm{~nm}$ and $850 \mathrm{~nm}$ wavelength, which correspond to the superposition of the complex optical resonances in the plasmonic semi-shells induced by their geometrical asymmetry. The nanodomes exhibit two different optical configurations, for the light polarized parallel to the longitudinal or transversal orientations of the nanodomes (see Fig. 2b). In the longitudinal configuration there are two main resonances of magnetic dipolar and quadrupolar character, located in the NIR and in the red part of the spectrum, respectively (Fig. S4). In contrast, two electric dipolar and quadrupolar resonances are observed in the transversal configuration that are blue shifted with respect to those in the longitudinal orientation (Fig. S4). Since the dispersed nanodomes are randomly distributed, the experimental spectrum is the convolution of these resonances averaged over all the possible orientations. The resonance band in the NIR region suites perfectly within the spectral region with higher penetration in physiological tissues 
(biological window) and, therefore, has high potential for photothermal applications. This resonance can be red-shifted even further by reducing the Au thickness (Fig. S5).
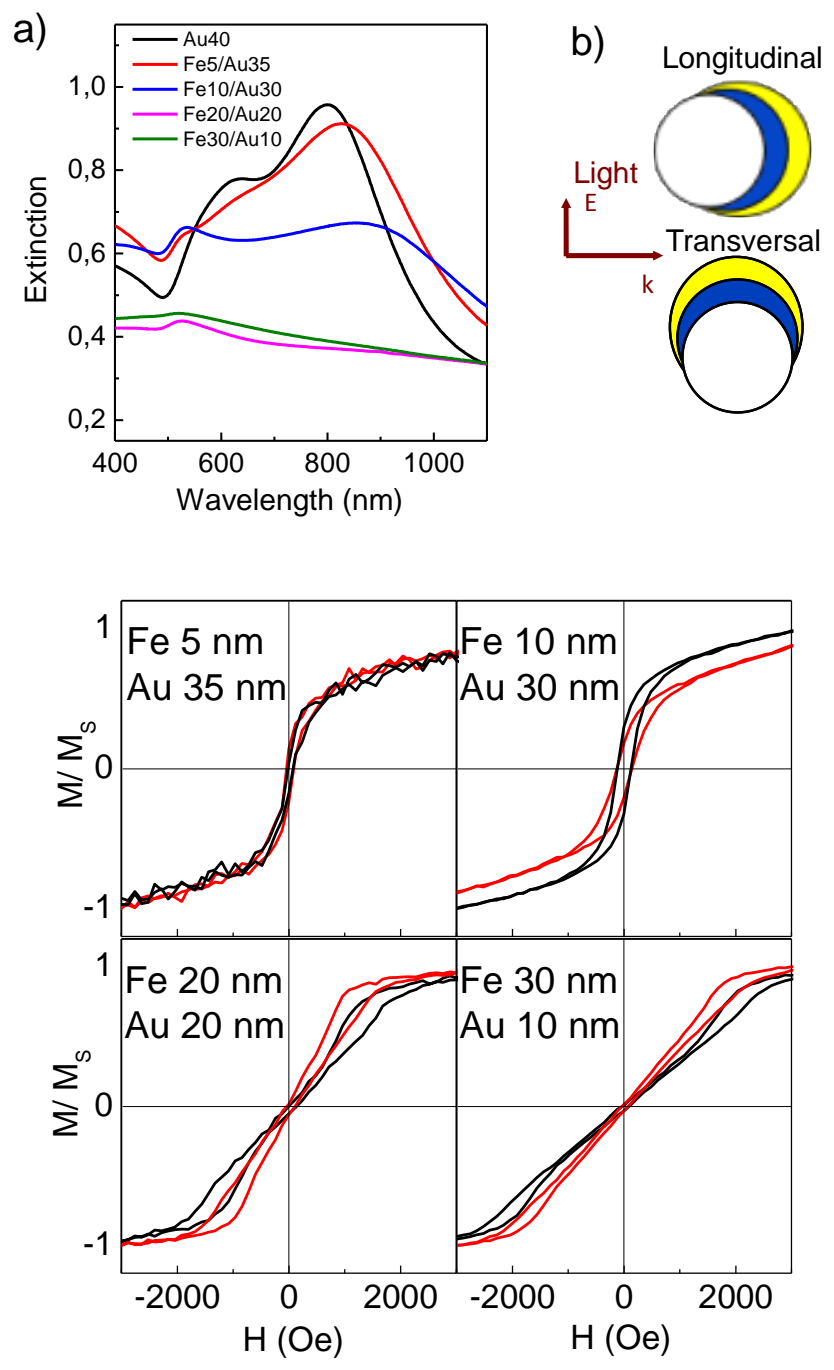

Fig. 2. Optical and magnetic properties of the MP nanodomes. a) Experimental vis-NIR spectra for MP nanoparticles with different Fe and Au ratios. b) Schematic of the two main optical configurations of the MP nanodomes, i.e. longitudinal and transversal configurations. c) Vibrating sample magnetometer magnetization loops of MP nanodomes with different $\mathrm{Fe}$ and $\mathrm{Au}$ ratios for in-plane (black) and out-of-plane (red) applied fields. 
The introduction of the Fe layer and the reduction of the Au thickness generate remarkable spectral changes in the nanodomes. A $5 \mathrm{~nm}$ thick Fe layer red shifts and broadens the NIR resonant band, and blue shifts the band initially centered at $650 \mathrm{~nm}$. As the Fe thickness increases, a substantial peak broadening and reduction of the extinction values are observed. For nanodomes with $20 \mathrm{~nm}$ Fe thickness and above, the plasmonic bands practically disappear, showing broadband extinction spectra with reduced extinction intensity. Such extinction reduction could initially suggest that the optical heating efficiency of MP nanodomes with high Fe content could substantially decrease. However, it has to be taken into account that the extinction measurements are the sum of scattering and absorption. In fact, finite difference time domain (FDTD) calculations in Fig. S4 show that increasing Fe thickness drastically reduces the scattering cross section of the nanodomes in both longitudinal and transversal orientations, nevertheless, the decrease in the absorption cross section is considerably weaker. Consequently, since the optical heating is only related to absorption and not to scattering, high optical heating efficiency can be still expected even with high Fe ratios, as we demonstrate below.

Interestingly, the MP nanodomes also offer tunable ferromagnetic properties by modifying the Fe thickness. Vibrating sample magnetometer measurements show that the MP nanodomes exhibit a ferromagnetic behavior at room temperature in all the studied Fe thicknesses (Fig. 2c). Nanodomes with $5 \mathrm{~nm}$ Fe thickness present single domain-like hysteresis loop with small coercitivity, which substantially increases for the $10 \mathrm{~nm}$ Fe thickness. Interestingly, a magnetic vortex is formed in the nanodomes with $20 \mathrm{~nm}$ and $30 \mathrm{~nm}$ Fe thickness showing near zero remanence hysteresis loops, as expected from the size and thickness of the Fe layer [35,36]. This magnetic behavior explains the observed high colloidal stability in all the MP nanodomes, even for high Fe content. Namely, when the magnetic vortex is formed, the magnetostatic interaction 
in the absence of external magnetic field is negligible. On the other hand, the magnetic dipoledipole interactions of the nanodomes with $5 \mathrm{~nm}$ and $10 \mathrm{~nm}$ Fe thickness are drastically reduced due to the large thickness of the Au layer and the strong electrostatic repulsion between particles.

We have analyzed the photothermal response of MP nanodomes for the different Fe/Au ratios, with concentrations ranging from $3 \cdot 10^{9}$ up to $1.2 \cdot 10^{11}$ nanodomes $/ \mathrm{mL}$ (Fig. 3b). A typical heating curve is shown in Fig. 3a, which displays the temperature rise in the nanodomes suspension when the laser is switched on, until the thermal equilibrium, due to the equal balance of absorbed and dissipated energy by the sample, is reached. The suspension slowly recovers the initial room temperature level once the laser is switched off.
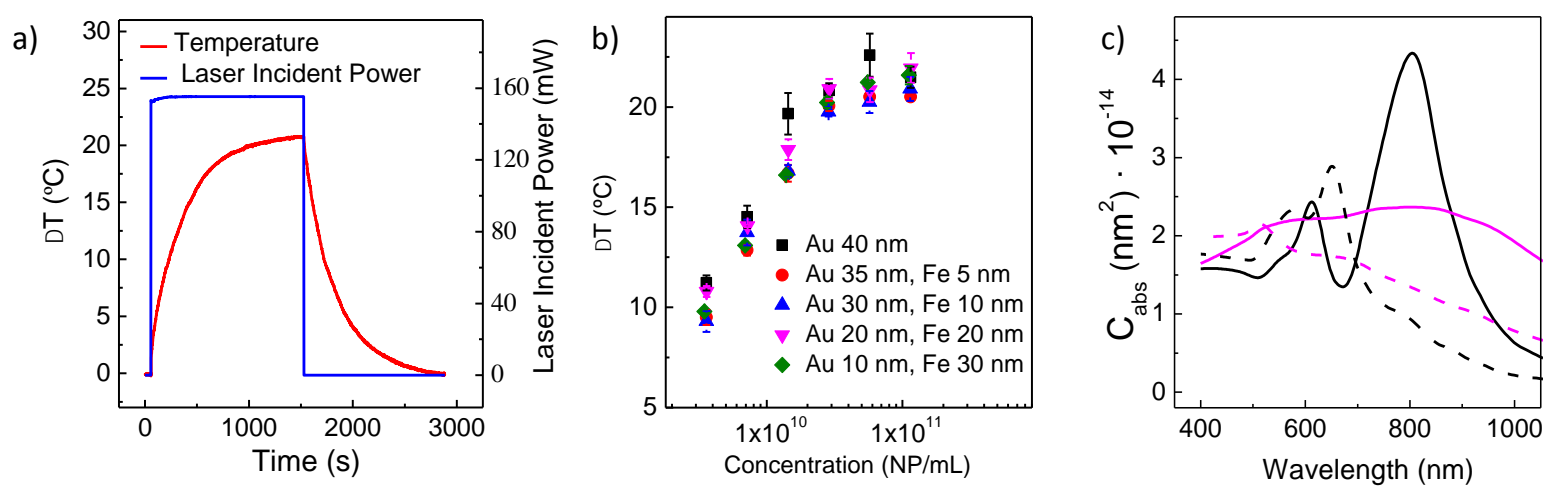

Fig. 3. Photothermal analysis. a) Example of laser heating experiment showing the temperature increase, $\Delta \mathrm{T}$, and decrease when the incident laser power is on or off, respectively. b) Temperature increase for MP nanodomes with different Fe and Au ratios and different concentrations (166 $\mathrm{mW}$ of incident laser power). c) Theoretical absorption cross-section of $\mathrm{Au} 40 \mathrm{~nm}$ (black) and $\mathrm{Au} 20 \mathrm{~nm} / \mathrm{Fe} 20 \mathrm{~nm}$ nanodomes (magenta) when the incident light polarization is in the longitudinal (continuous lines) or transversal (dashed lines). 
As expected, the temperature increase follows a linear dependence with the colloidal concentration for low concentration levels. However, the temperature increase saturates at a concentration of $3 \cdot 10^{10}$ nanoparticles $/ \mathrm{mL}$ due to the complete absorption of the laser light along the $1 \mathrm{~cm}$ thick cuvette for higher nanodomes concentrations. Nevertheless, the most remarkable result is the almost identical optical heating efficiency for all nanodome configurations, regardless of the Fe/Au ratio (Fig. 3b). The reason behind this striking behavior can be inferred from Fig. 3c. Gold nanodomes are highly anisotropic structures showing four-fold difference in their absorption cross section for the longitudinal and transversal configurations at $800 \mathrm{~nm}$ wavelength. Consequently, only Au nanodomes that are longitudinally oriented with respect to the incident light polarization are efficiently heated. In contrast, the optical anisotropy is reduced as the Fe content increases, as Fig. 3c shows for the $20 \mathrm{~nm}$ of Fe and $20 \mathrm{~nm}$ of Au nanodomes. Interestingly, the decrease in the absorption efficiency of $\mathrm{Fe} / \mathrm{Au}$ nanodomes with respect to $\mathrm{Au}$ nanodomes in the longitudinal configuration is partially compensated by a higher efficiency in the transversal configuration. In addition, the heating efficiency of nanodomes with high $\mathrm{Fe}$ content benefits from their low scattering cross section (Fig. S4), thereby minimizing the backscattered radiation that does not contribute to nanodomes heating. As a result, all the MP nanodomes achieve similar temperature increments for a given laser power and particle concentration. Interestingly, even at rather low particle concentrations (in the $10^{9}$ nanodomes $/ \mathrm{mL}$ range), temperature increments for therapeutic applications (from $5{ }^{\circ} \mathrm{C}$ to $8{ }^{\circ} \mathrm{C}$ ) can be easily achieved. At this point, it is worth analyzing the photothermal conversion efficiency of the MP nanodomes with different $\mathrm{Fe} / \mathrm{Au}$ ratios compared to state-of-the-art plasmonic nanoheaters, such as $\mathrm{Au}$ nanorods and $\mathrm{Au}$ nanoshells showing plasmonic resonances close to $810 \mathrm{~nm}$. The photothermal conversion efficiency, $\eta$, is calculated by [37]: 


$$
\eta=\frac{h S \Delta T_{\max }-Q_{d i s}}{I\left(1-10^{-A_{808}}\right)}
$$

where $h$ is the heat transfer coefficient, $S$ is the laser irradiating area, $\Delta T_{\max }$ is the optically induced temperature change when the thermal equilibrium is reached, $Q_{d i s}$ is the heat dissipation from the experimental set-up, $I$ is incident laser power $(166 \mathrm{~mW})$, and $A_{808}$ is the absorbance of the nanoparticles at $808 \mathrm{~nm}$. The value $h S$ is given by:

$$
h S=\frac{m_{\text {water }} C_{\text {water }}}{\tau_{S}}
$$

where $m_{\text {water }}$ and $C_{\text {water }}$ are the mass and heat capacity of deionized water $(0.4 \mathrm{~g}$ and $4.2 \mathrm{~J} / \mathrm{g}$, respectively), and $\tau_{s}$ is the set-up time constant $(250 \mathrm{~ms})$, i.e. the time required to recover a temperature increase equal to $\left(\Delta T_{\max } / e\right)$ in the set up once the light source is switched off and the sample cools down. The value of $Q_{\text {dis }}(3.36 \mathrm{~mW})$ was measured using a plastic cuvette containing deionized water $(400 \mu \mathrm{L})$. The photothermal efficiency results are gathered in Table 1, together with the $A_{808}$ and $\Delta T_{\max }$ values experimentally obtained from the different nanoparticle dispersions.

\begin{tabular}{|l|c|c|c|}
\cline { 2 - 4 } \multicolumn{1}{c|}{} & $A_{808}$ & $\Delta T_{\max }$ & $\boldsymbol{\eta}$ \\
\hline Au 40 nm NDs & 0.45 & 9.5 & $\mathbf{0 . 5 6}$ \\
\hline Fe 20nm/Au 20 nm ND & 0.54 & 12.0 & $\mathbf{0 . 6 5}$ \\
\hline Au nanorods & 0.74 & 14.0 & $\mathbf{0 . 6 7}$ \\
\hline Au nanoshells & 0.56 & 6.1 & $\mathbf{0 . 3 1}$ \\
\hline
\end{tabular}

Table 1. Values of the photothermal conversion efficiency of the $\mathrm{Au}$ and $\mathrm{Fe} / \mathrm{Au}$ nanodomes compared to Au nanorods and Au nanoshells.

$$
\eta=\frac{\mathrm{hS}\left(\mathrm{T}_{\max }-\mathrm{T}_{\mathrm{Surr}}\right)-\mathrm{Q}_{\mathrm{dis}}}{\mathrm{I}\left(1-10^{\left.-\mathrm{A}_{808}\right)}\right.}(1)
$$


As can be observed in Table 1, the Fe/Au nanodomes efficiency is clearly higher than that of $\mathrm{Au}$ nanodomes and is equal to that of the Au nanorods, which are the most efficient plasmonic nanoheaters. Such a high efficiency in the Fe/Au nanodomes is due their minimized scattering cross section of the Fe/Au nanodomes and low optical anisotropy, as it was discussed below. The combination of both effects enables a deeper light penetration and a more uniform heating of the colloidal dispersion in the case of Fe/Au nanodomes. In addition, the heating efficiency is much larger than that of nanoshells, which are nanostructures that exhibit a large scattering cross section, and lower absorption cross section in the near infrared.

In addition to a higher photothermal conversion efficiency, the $\mathrm{Fe}$ layers confer the nanodomes with the unique capacity to magnetically control and amplify photothermal therapies. To experimentally assess such ability, we have first analyzed their magnetic trapping efficiency via magnetophoretic forces, by attaching a cylindrical FeNdB magnet $(6 \mathrm{~mm}$ diameter, $10 \mathrm{~mm}$ length, with a $2.5 \mathrm{kOe}$ field at the surface) at the lateral side of the cuvette that is parallel to the light path (see Fig. S2). To compare the magnetophoretic forces among nanodomes, we have quantified the time that is required to achieve the $95 \%$ of the transmitted laser power (taking $100 \%$ as a water sample without particles), as a method to determine when the majority of the nanodomes are magnetically trapped at the cuvette wall. Magnetic trapping takes more than $1 \mathrm{~h}$ for nanodomes with only $5 \mathrm{~nm}$ of Fe. In contrast, the trapping time is reduced to $6 \mathrm{~min}$ for $10 \mathrm{~nm}$ Fe nanodomes, and less than $2 \mathrm{~min}$ for $20 \mathrm{~nm}$ and $30 \mathrm{~nm}$ Fe nanodomes (Fig. 4a). The large trapping time for $5 \mathrm{~nm}$ Fe nanodomes is due to their weak magnetic dipole moment and large mass given by the thick of $\mathrm{Au}$ layer. Increasing the $\mathrm{Fe}$ content to $10 \mathrm{~nm}$, induces a 9-fold enhancement of the nanodomes magnetization (Fig. S6), probably due to a reduced magnetization at the $\mathrm{Fe} / \mathrm{Au}$ interface $[38,39]$, whose net magnetic effect is more pronounced for 
thin Fe films. The larger mass of the $5 \mathrm{~nm} \mathrm{Fe} \mathrm{nanodomes} \mathrm{and,} \mathrm{consequently,} \mathrm{their} \mathrm{lower}$ acceleration caused by the magnetic force can account for the 11-fold rise of the trapping time of $5 \mathrm{~nm}$ thick Fe nanodomes compared to that of $10 \mathrm{~nm}$ thick Fe. The increase of the Fe thickness to $20 \mathrm{~nm}$ and $30 \mathrm{~nm}$ induce an additional 4-fold reduction of the trapping time. Such reduction is the result of the larger magnetic moment and lower mass of the nanodomes with high Fe ratio. The only slight reduction of the trapping time in the $30 \mathrm{~nm}$ Fe nanodomes compared to that in the $20 \mathrm{~nm}$ Fe nanodomes is due to the more tilted hysteresis loop in the former ones (see Fig. 2c), which compensates their higher Fe content and lower mass.
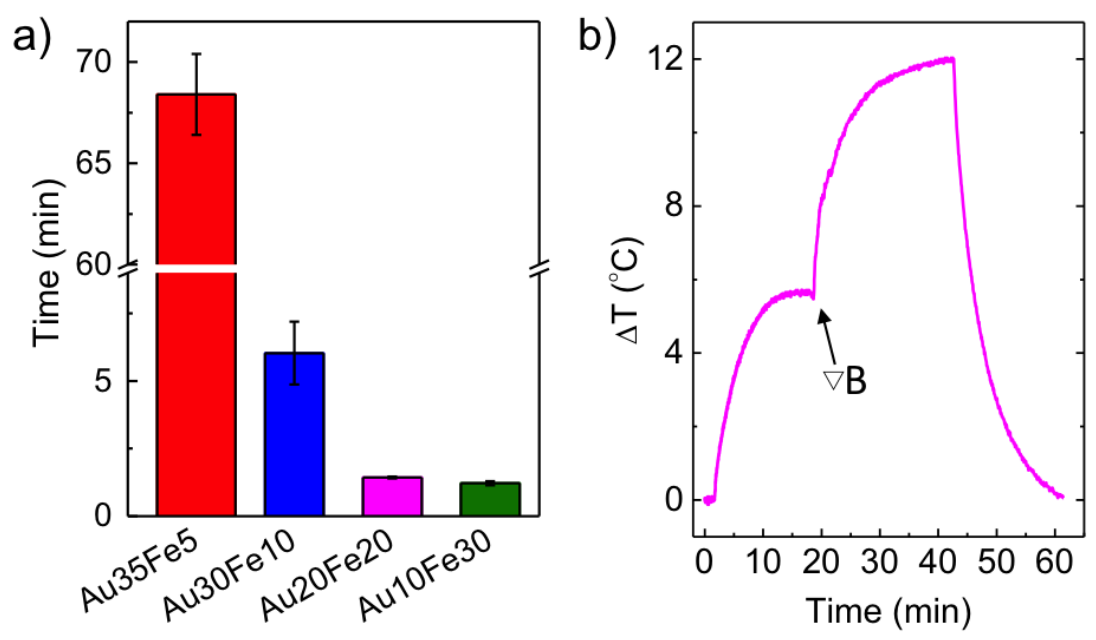

Fig. 4. Magnetic actuation and photothermal amplification analysis. a) Time required to achieve 95\% light transmission for MP nanodomes with different $\mathrm{Fe}$ and Au ratios due to the magnetic trapping by a permanent magnet that is attached to the cuvette wall. b) Temperature increment amplification by magnetically increasing the particle concentration of the Fe $20 \mathrm{~nm} / \mathrm{Au} 20 \mathrm{~nm}$ nanodomes in the laser path. The initial particle concentration is $2.4 \cdot 10^{9}$ particles $/ \mathrm{mL}$. 
To highlight the strength of the magnetic manipulation it is worth comparing the magnetic dipole $(m)$ that can be generated in the nanodomes and in standard colloidally stable SPIONs. The maximum magnetic dipole that can be generated in the nanostructures is given by $m=M_{S}$ $V_{P}$, where $M_{S}$ is the saturation magnetization and $V_{P}$ is the volume of the magnetic element. In the case of nanodomes with $20 \mathrm{~nm}$ Fe thickness, the maximum magnetic dipole is $c a$. 630-fold larger than that of FDA (US Food and Drug Administration)-approved SPIONs with $12 \mathrm{~nm}$ diameter, and almost three orders of magnitude larger for Fe $30 \mathrm{~nm}$ nanodomes. This huge difference is due to the 3 -fold higher $M_{S}$ of metallic iron compared to that of iron oxide (i.e., $1716 \mathrm{emu} / \mathrm{cm}^{3}$ for $\mathrm{Fe}$ versus $476 \mathrm{emu} / \mathrm{cm}^{3}$ for $\mathrm{Fe}_{3} \mathrm{O}_{4}$ ) and the much larger volume of the $\mathrm{Fe}$ layer in the nanodome. As a result, the nanodomes can act as very strong nanomagnets in the presence of a magnetic field (thus, reacting quickly to them), although they can keep high colloidal stability in the absence of magnetic fields due to their near zero remanence (given by their magnetic vortex state). Actually, mild sonication can easily disperse the magnetically trapped nanodomes and fully recover the homogeneous colloidal dispersion once the magnet is removed.

Interestingly, the efficient magnetophoretic manipulation can be used to locally amplify the optical heating efficiency by increasing the particle concentration at the illumination region. This effect is demonstrated for Fe $20 \mathrm{~nm} / \mathrm{Au} 20 \mathrm{~nm}$ nanodomes in Fig. 4b, in which an 85\% enhancement in the temperature rise is observed when particles are magnetically concentrated at the region that blocks the laser path in the cuvette wall (see Fig. S2). As we show below, this effect is especially valuable to locally enhance the photothermal treatments for efficiently killing tumor cells.

In addition to the significant fabrication cost reduction with respect to pure plasmonic nanoparticles and the magnetically enhanced photothermal effects, the Fe layer in the MP 
nanodomes provide an intense NMR contrast for imaging. To maximize the NMR signal we focus the NMR analysis on the MP nanodomes with the largest magnetic dipole, i.e. $30 \mathrm{~nm}$ of Fe. We have studied the relaxation rates $\left(R_{1}=1 / T_{1}\right.$ and $\left.R_{2}=1 / T_{2}\right)$ as a function of the Fe molar concentration in samples of MP nanoparticles dispersed in agar gel (Fig. 5a). While we do not observe any significant $T_{1}$ contrast, which is a consequence of the presence of the diamagnetic Au layer in contact to water molecules, we detect very high $T_{2}$ contrast. The slope of the $\mathrm{R}_{2}$ curve as a function of $\mathrm{Fe}$ concentration reveals that the relaxivity $r_{2}$ is $266 \pm 27 \mathrm{mM}^{-1} \mathrm{~s}^{-1}$. This relaxivity is between 1.5 - and 2-fold larger than that of superparamagnetic iron oxide nanoparticles that have been commercially available as $T_{2}$ contrast agents, like Feridex $\left(105 \mathrm{mM}^{-}\right.$ ${ }^{1} \mathrm{~s}^{-1}$ at $\left.47 \mathrm{kOe}\right)$ or Resovist $\left(176 \mathrm{mM}^{-1} \mathrm{~s}^{-1}\right.$ at $\left.47 \mathrm{kOe}\right)$ [40]. Even though these values correspond to slightly lower fields than in our case, substantial increase in their $T_{2}$ from $47 \mathrm{kOe}$ to $70 \mathrm{kOe}$ is not expected [40]. Therefore, compared to, for example, the Resovist nanoparticles (50 $\mathrm{nm}$ diameter), the larger size and higher density of metallic iron in the MP nanodomes enable packing a much higher amount of Fe atoms per particle. In addition, the Fe atoms in a metallic state induce higher relaxivity than oxidized iron atoms. The sum of both effects makes that the net relaxivity per particle of MP nanodomes is approximately 15 -fold higher than that of Resovist particles, highlighting their high potential as $\mathrm{T}_{2}$ NMR contrast agent. Notably, the nanodomes concentration range necessary to observe sizable $\mathrm{T}_{2}$ effects is similar to that required for the photothermal treatments, which paves the way for efficient NMR-imaging guided photothermal therapies.

Moreover, the Au layer in the MP nanodomes is a very efficient contrast agent for X-Ray imaging. We have exploited this capacity to visualize the internalized nanodomes by synchrotron-based soft X-ray transmission microscopy operating in the water window energy 
range $(520 \mathrm{eV})$. By employing cryogenic conditions, the soft X-ray microscope enables operating in an environment close to the hydrated physiological conditions. Thus, soft X-ray microscopy can also yield 3D structural information of the entire cell without the need of fixation, dehydration, embedding and sectioning of the samples. The resolution of the soft X-ray microscope is about $40 \mathrm{~nm}$, which is sufficient to visualize individual MP nanodomes at different cellular planes and to map their interaction with the cellular compartments. The X-ray images of Fig. $5 \mathrm{~b}$ clearly show the individual MP nanodomes internalized by the cells. The movie included in the supporting information enables visualizing the MP nanodomes at different z-planes inside the cell. In addition, for reference, Fig. S9 displays some nanodomes which are outside the cells, since they are added after the cell freezing process to help in the image reconstruction process, showing similar morphology and X-ray absorption. These results highlight the potential of the MP nanodomes as contrast agents in X-ray computed tomography. 
a)

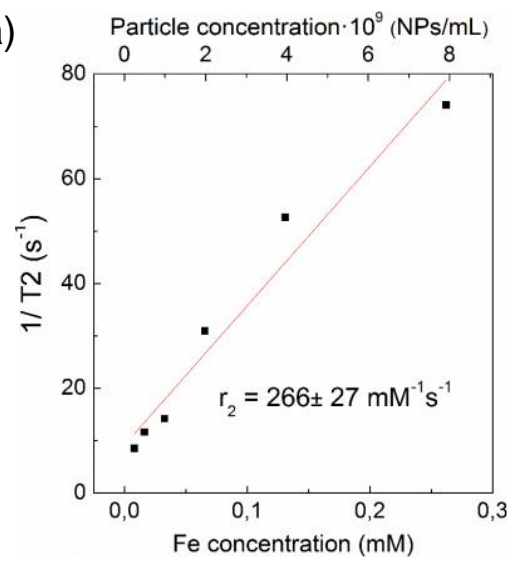

c)

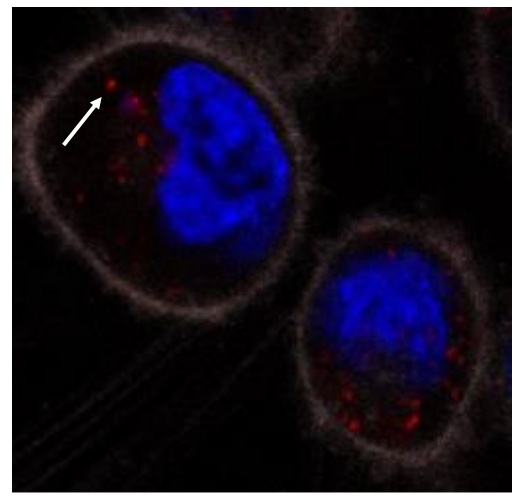

b)

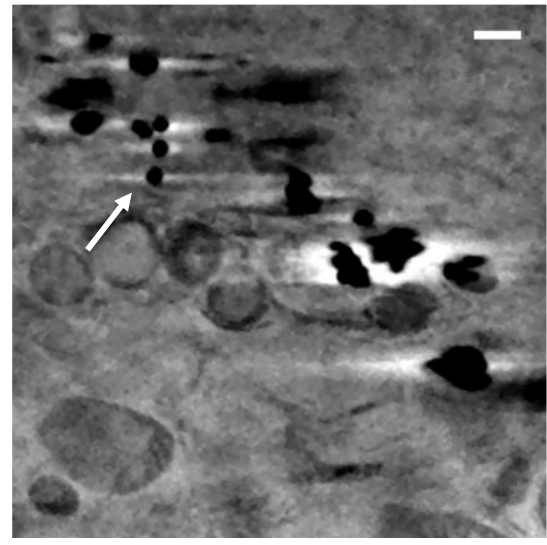

d)

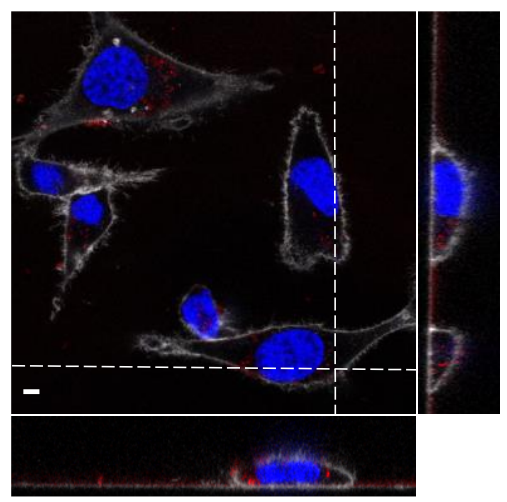

Fig. 5. MP nanodomes as contrast agents for imaging a) NMR $R_{2}\left(1 / T_{2}\right)$ values of the MP nanodomes with Fe $30 \mathrm{~nm} / \mathrm{Au} 10 \mathrm{~nm}$ in agar gel as a function of Fe concentration. b) Transmission X-Ray microscopy image showing the internalized Fe $20 \mathrm{~nm} / \mathrm{Au} 20 \mathrm{~nm} \mathrm{MP}$ nanodomes as black dots (white arrow). The black diffused regions correspond to nanodomes in different focal planes (see Video S1). c) Confocal optical image of HeLa cells that have internalized Fe $20 \mathrm{~nm} / \mathrm{Au} 20 \mathrm{~nm}$ MP nanodomes with fluorescent core, seen as red dots in the image (white arrow). The plasma membranes are shown in white and the nuclei in blue. d) Confocal image (middle) and its orthogonal projections of the z-stack reconstructions (right and bottom) of consecutive focal planes $(0.5 \mu \mathrm{m}$ each $)$. Discrete red spots corresponding to MP nanodomes trapped in endosomes/lysosomes can be observed. Scale bars: $5 \mu \mathrm{m}$. 
Further, MP nanodomes with fluorescent cores provide additional valuable imaging functionalities to understand their in vitro behavior and internalization pathways. We have integrated this functionality by fabricating the nanodomes on fluorescent polystyrene nanobeads (excitation at $570 \mathrm{~nm}$ and emission at $610 \mathrm{~nm}$ ), yielding similar surface density on the wafer and colloidal stability. We have employed Fe $20 \mathrm{~nm} / \mathrm{Au} 20 \mathrm{~nm}$ nanodomes with fluorescent cores to visualize their interaction and internalization by HeLa cancer cells via 3D confocal microscopy reconstructions and cross-section projections (Figs. $5 \mathrm{c}$ and d). Although the Fe/Au bilayer induces a 5-fold reduction of the fluorescent intensity with respect to the bare polymer particles, due to the high absorption cross section of the nanodomes, the fluorescent Fe/Au nanodomes can still be easily visualized by confocal microscopy. To discern internalized from not internalized MP nanodomes, the plasma membrane was labeled (white) to establish the cell boundaries. The nuclei were stained (blue) to determine whether MP nanodomes were able to translocate to the nucleus. Images obtained at different focal plane confirmed that, in the absence of magnetic field, the MP nanodomes could be fully internalized, rather than resting on the cell surface, as it can be seen in the orthogonal cross-sections (Fig. 5d). Discrete red spots, corresponding to MP nanodomes trapped in endosomes/lysosomes can be observed inside the cytoplasm, but not inside the nucleus due to their large size (100 nm in diameter), which prevents crossing the nuclear porous complex [41]. Probably, MP nanodomes are internalized via pinocytosis, forming endosomes that can be visualized as discrete points.

To assess the biomedical potential of the MP nanodomes, we finally tested the effect of photothermal treatments in HeLa cells in challenging conditions of low particle concentration and mild laser intensity (Fig. 6). We used glass bottom dishes with a thickness of $0.17 \mathrm{~mm}$ to facilitate light irradiation, where cells were seeded only in the glass region. Cells were incubated 
for $3 \mathrm{~h}$ with cell culture medium containing a concentration of either $10 \mu \mathrm{g} / \mathrm{mL}$ or $100 \mu \mathrm{g} / \mathrm{mL}$ of Fe $20 \mathrm{~nm} / \mathrm{Au} 20 \mathrm{~nm}$ nanodomes.
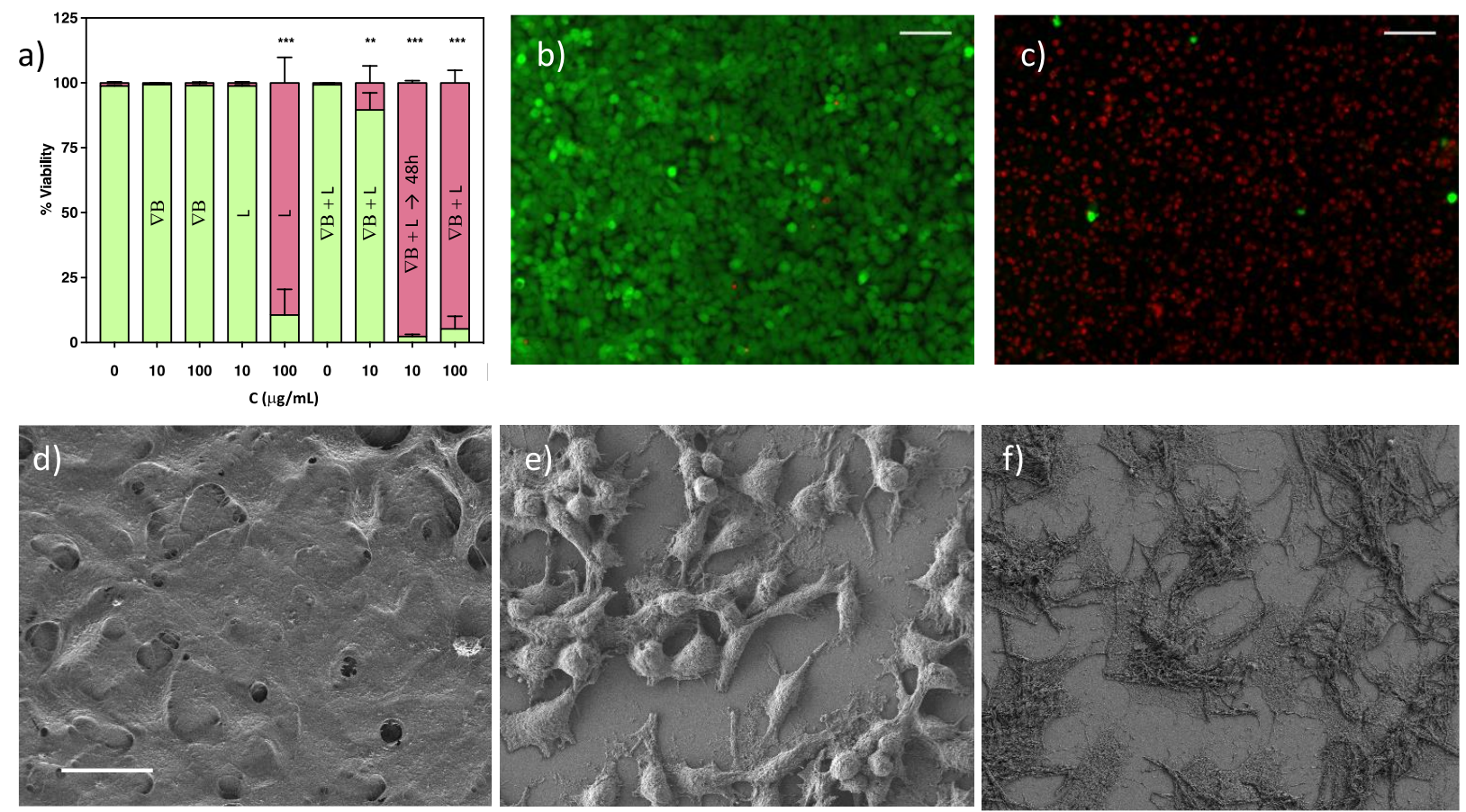

Fig. 6. Effects of photothermal treatment in vitro. a) Results of the in vitro photothermal treatments in HeLa cells under different concentrations of MP nanodomes (C), magnetic manipulation ( $\nabla \mathrm{B})$ and light actuation $(\mathrm{L})$ conditions. The viability assays were performed $24 \mathrm{~h}$ after the treatment, expect for the nanodomes with concentration of $10 \mu \mathrm{g} / \mathrm{mL}$ under magnetic manipulation and light treatment $(\nabla \mathrm{B}+\mathrm{L})$, in which the assays were carried out both after $24 \mathrm{~h}$ and $48 \mathrm{~h} . \mathrm{b}$ and c) Typical fluorescent images of live (green) and dead (red) cells of a sample with non-irradiated cells, and a sample of cells after the light treatment, respectively, for an initial concentration of $10 \mu \mathrm{g} / \mathrm{mL}$ of nanodomes with magnetic concentration (scale bars $100 \mu \mathrm{m}$ ). d)-f) Scanning electron microscopy images of the HeLa cells for the control (d), and after the magnetic concentration and light treatment for initial nanodomes concentrations of $10 \mu \mathrm{g} / \mathrm{mL}$ (e) and $100 \mu \mathrm{g} / \mathrm{mL}$ (f). Scale bar: $20 \mu \mathrm{m}$. 
We have compared the photothermal treatment efficiency either with or without magnetic field concentration of the nanodomes at the illumination region. After the incubation time, the cell culture medium with nanoparticles was replaced by $1 \mathrm{~mL}$ of fresh medium to leave only the particles in contact or inside the cells. The cell monolayer was irradiated with a laser at $808 \mathrm{~nm}$ emission wavelength during $30 \mathrm{~min}$ with an incident intensity of $5 \mathrm{~W} / \mathrm{cm}^{2}$. Due to the limited irradiated area (laser spot diameter of $6 \mathrm{~mm}$ ), we analyzed cell viability using a colorimetric assay (LIVE/DEAD ${ }^{\circledR}$ ), which enables quantifying live (green) and dead (red) cells according to their esterase activity and membrane integrity, respectively (Fig. 6 a-c). In these assays we compared the light treatment effect in control samples without nanodomes and in samples with two different initial concentrations of nanodomes $(10 \mu \mathrm{g} / \mathrm{mL}$ and $100 \mu \mathrm{g} / \mathrm{mL})$, either in the absence of magnetic field or with magnetic concentration at the illumination region by a spherical FeNdB magnet (12 mm in diameter, $2.5 \mathrm{kOe}$ at the surface) (see Fig. S8). Firstly, the results show that irradiated cell cultures without MP nanodomes do not exhibit statistically significant differences with the non-irradiated control (Fig. 6a), i.e. the laser actuation does not cause cytotoxicity. Secondly, we evaluated the effect of the MP nanodomes in cell cultures with and without magnetic concentration, showing that the presence of MP nanodomes does not induce a significant viability decreases, even for high nanodomes concentration $(100 \mu \mathrm{g} / \mathrm{mL})$ under magnetic concentration conditions (Fig. 6a). Finally, we assessed the effect of light irradiation in cells containing MP nanodomes. The results show a minimal decrease in the cell viability without magnetic concentration for a nanodomes concentration of $10 \mu \mathrm{g} / \mathrm{mL}$. The concentration must be increased up to $100 \mu \mathrm{g} / \mathrm{mL}$ to observe a significant viability reduction in the absence of magnetic concentration. In contrast, a viability reduction of nearly $100 \%$ after the 
light treatment is observed in the case of magnetic concentration at the illumination region for both initial nanodomes concentrations of $10 \mu \mathrm{g} / \mathrm{mL}$ and $100 \mu \mathrm{g} / \mathrm{mL}$ (Fig. 6a). Interestingly, such drastic viability reduction is observed $24 \mathrm{~h}$ after the treatment for the initial $100 \mu \mathrm{g} / \mathrm{mL}$ concentration, whereas it takes $48 \mathrm{~h}$ to get the near $100 \%$ reduction when the initial concentration is $10 \mu \mathrm{g} / \mathrm{mL}$. The different light effect on the cancer cells can be clearly observed in the scanning electron microscopy images of Fig. 6 d-f. For magnetically concentrated samples with initial $100 \mu \mathrm{g} / \mathrm{mL}$ concentration, a clear loss of cell membrane integrity is observed, thereby reflecting the unfavorable fast necrotic cell death that is caused by the very intense photothermal actuation under these conditions. On the contrary, for the initial concentration of $10 \mu \mathrm{g} / \mathrm{mL}$, the cell membrane disruption is not perceived, although there is a clear morphological change in the cancer cells. The longer time to generate the viability reduction suggests a more controlled cell death pathway, which is desirable for the photothermal treatments. Importantly, the magnetic concentration at the light treatment region enables at least a 10 -fold reduction of the quantity of injected particles to achieve the nearly $100 \%$ therapeutic efficacy, which can be highly relevant to reduce the therapy cost and the bioaccumulation of the nanoparticles in other organs.

The comparison of our results with other nanoparticles for photothermal therapies is not straightforward, since different composition, surface functionalization and concentration of particles have been employed, in addition to different irradiation conditions, and cell types [42]. Nevertheless, some trends can be found regarding particle concentration and exposure conditions. Studies that use high nanoparticle concentration (from 100 to $250 \mu \mathrm{g} / \mathrm{mL}$ ) apply low or mild exposure conditions (from 0.1 to $10 \mathrm{~W} / \mathrm{cm}^{2}$ ), while studies using low nanoparticles concentration (from 6.6 to $36.5 \mu \mathrm{g} / \mathrm{mL}$ ) typically need higher exposure conditions (from 15.3 to $250 \mathrm{~W} / \mathrm{cm}^{2}$ ) to obtain good efficiencies on cell death. In the present work, MP nanodomes have 
demonstrated to be a good candidate for photothermal treatments using low initial concentration $(10 \mu \mathrm{g} / \mathrm{mL})$ and mild incident intensity $\left(5 \mathrm{~W} / \mathrm{cm}^{2}\right)$ compared to other published studies [43]. This laser intensity is higher than the recommended clinical safety values $\left(0.3 \mathrm{~W} / \mathrm{cm}^{2}\right)$, but our in vitro assays with cell monolayers are much more demanding from the optical perspective than in vivo conditions, in which light can be efficiently absorbed along several millimeters. In contrast, the light absorption region is thinner than $10 \mu \mathrm{m}$ in a 2D cell culture with nanoparticles only in the cell monolayer. The generated heat in the cell monolayer rapidly diffuses towards the cell medium and the glass substrate due to the generated temperature difference between the cell monolayer and both the cell media and the substrate, thereby forcing the need of higher light intensity to achieve temperature increments with sufficient therapeutic effect.

Finally, it is worth mentioning that, although the magnetic manipulation is a powerful tool to locally control the photothermal effects, the nanodomes could offer additional cell targeting by exploiting their straightforward functionalization with specific antibodies, proteins such as transferrin (see Fig. S2), and molecules such as folate [44], musuin7 [41], RGD peptide [42] or other specific molecules capable of preferentially binding the MP nanodomes to cancer cells overexpressing particular membrane receptors.

\section{Conclusion}

We have shown that MP nanodomes can have high potential for therapeutic and diagnostic applications. Compared to other magnetic, plasmonic or magneto-plasmonic nanoparticles fabricated by chemical synthesis, our fabrication process enables easier control in the magnetic and optical properties. The capacity to functionalize the MP nanodomes on the substrate and their direct dispersion in water or buffer represent also significant advantages with respect to 
chemically synthesized nanoparticles, yielding highly stable colloidal dispersions during months. Compared to state-of-the-art plasmonic nanoparticles for photothermal therapies, MP nanodomes offer higher heating efficiency at lower cost given by their strong manipulation via magnetophoretic force, very high contrast for NMR and X-ray imaging, and easy incorporation of fluorophores in the polymer core. Compared to iron oxide nanoparticles used in magnetic hyperthermia, the high heating efficiency of MP nanodomes enables local thermal treatments at much lower particle concentration [45]. Moreover, MP nanodomes show much higher $T_{2}$ contrast per particle for NMR imaging than that of commercial iron oxide nanoparticles. Demonstration of both low cytotoxicity and magnetically enhanced efficiency for photothermal therapy at low particle concentrations and mild light intensity encourages the transfer of this nanotechnology to in vivo therapies. The high optical heating efficiency of the nanodomes could be also applied to develop temperature responsive drug delivery systems that could be magnetically controlled and visualized via computed X-ray tomography, NMR imaging, or fluorescence. The optical anisotropy in nanodomes with low Fe ratio and their capacity to efficiently rotate in the liquid under an alternating magnetic field can also provide interesting tools for the development of nanobiosensors and nanothermometers [46]. 


\section{Acknowledgements}

Zhi Li and Antonio Aranda-Ramos contributed equally to this work. This work is supported by Spanish Ministry of Economy, Industry and Competitiveness (MINECO) and European Regional Development Fund (FEDER) (Projects number: MAT2013-48628-R, MAT2014-57960-C3-1-R, MAT2016-77391-R, MAT2017-86357-C3-1-R and MAT2017-86357-C3-3-R). Generalitat de Catalunya (2017-SGR-292) is acknowledged. ICN2 is funded by the Centres de Recerca de Catalunya(CERCA) Programme / Generalitat de Catalunya. ICN2 also acknowledges support from the Severo Ochoa Program (MINECO, Grant SEV-2013-0295). Zhi Li acknowledges the Chinese Scholarship Council Program (201506950059) for financial support. The ALBA Synchrotron is acknowledged for the provision of beamline, and the MISTRAL beamline staff is acknowledged for their help during the X-ray microscopy measurements. Jordi Sort acknowledges the financial support by the European Research Council (SPIN-PORICS 2014Consolidator Grant, Agreement No 648454).

\section{References}

[1] G.J. Kim, S. Nie, Targeted cancer nanotherapy, Mater. Today. 8 (2005) 28-32. doi:10.1016/S1369-7021(05)71034-8.

[2] R. Bardhan, S. Lal, A. Joshi, N.J. Halas, Theranostic nanoshells: From probe design to imaging and treatment of cancer, Acc. Chem. Res. 44 (2011) 936-946. doi:10.1021/ar200023x.

[3] S.D. Jo, S.H. Ku, Y.Y. Won, S.H. Kim, I.C. Kwon, Targeted nanotheranostics for future personalized medicine: Recent progress in cancer therapy, Theranostics. 6 (2016) 13621377. doi:10.7150/thno.15335.

[4] G. Chen, I. Roy, C. Yang, P.N. Prasad, Nanochemistry and Nanomedicine for Nanoparticle-based Diagnostics and Therapy, Chem. Rev. 116 (2016) 2826-2885. doi:10.1021/acs.chemrev.5b00148.

[5] X.H. Huang, I.H. El-Sayed, W. Qian, M.A. El-Sayed, Cancer cell imaging and photothermal therapy in the near-infrared region by using gold nanorods, J. Am. Chem. Soc. 128 (2006) 2115-2120. doi:10.1021/ja057254a.

[6] S. Lal, S.E. Clare, N.J. Halas, Nanoshell-enabled photothermal cancer therapy: Impending clinical impact, Acc. Chem. Res. 41 (2008) 1842-1851. doi:10.1021/ar800150g.

[7] C.M. Hessel, V. P. Pattani, M. Rasch, M.G. Panthani, B. Koo, J.W. Tunnell, B.A. Korgel, Copper selenide nanocrystals for photothermal therapy, Nano Lett. 11 (2011) 2560-2566. doi:10.1021/nl201400z.

[8] L. Cheng, K. Yang, Y. Li, J. Chen, C. Wang, M. Shao, S.T. Lee, Z. Liu, Facile preparation of multifunctional upconversion nanoprobes for multimodal imaging and dual-targeted photothermal therapy, Angew. Chemie - Int. Ed. 50 (2011) 7385-7390. doi:10.1002/anie.201101447.

[9] Z. Sun, H. Xie, S. Tang, X.F. Yu, Z. Guo, J. Shao, H. Zhang, H. Huang, H. Wang, P.K. Chu, Ultrasmall Black Phosphorus Quantum Dots: Synthesis and Use as Photothermal Agents, Angew. Chemie - Int. Ed. 54 (2015) 11526-11530. doi:10.1002/anie.201506154. 
[10] W. Zhang, Z. Guo, D. Huang, Z. Liu, X. Guo, H. Zhong, Synergistic effect of chemophotothermal therapy using PEGylated graphene oxide, Biomaterials. 32 (2011) 85558561. doi:10.1016/j.biomaterials.2011.07.071.

[11] H. Liu, D. Chen, L. Li, T. Liu, L. Tan, X. Wu, F. Tang, Multifunctional gold nanoshells on silica nanorattles: A platform for the combination of photothermal therapy and chemotherapy with low systemic toxicity, Angew. Chemie - Int. Ed. 50 (2011) 891-895. doi:10.1002/anie.201002820.

[12] W. Guo, F. Wang, D. Ding, C. Song, C. Guo, S. Liu, TiO2-xBased Nanoplatform for Bimodal Cancer Imaging and NIR-Triggered Chem/Photodynamic/Photothermal Combination Therapy, Chem. Mater. 29 (2017) 9262-9274. doi:10.1021/acs.chemmater.7b03241.

[13] J.C. Yang, Y. Chen, Y.H. Li, X.B. Yin, Magnetic Resonance Imaging-Guided Multi-Drug Chemotherapy and Photothermal Synergistic Therapy with $\mathrm{pH}$ and NIR-Stimulation Release, ACS Appl. Mater. Interfaces. 9 (2017) 22278-22288. doi:10.1021/acsami.7b06105.

[14] A. Li Volsi, C. Scialabba, V. Vetri, G. Cavallaro, M. Licciardi, G. Giammona, NearInfrared Light Responsive Folate Targeted Gold Nanorods for Combined PhotothermalChemotherapy of Osteosarcoma, ACS Appl. Mater. Interfaces. 9 (2017) 14453-14469. doi:10.1021/acsami.7b03711.

[15] J.R. Cole, N.A. Mirin, M.W. Knight, G.P. Goodrich, N.J. Halas, Photothermal efficiencies of nanoshells and nanorods for clinical therapeutic applications, J. Phys. Chem. C. 113 (2009) 12090-12094. doi:10.1021/jp9003592.

[16] X. Huang, M.A. El-Sayed, Gold nanoparticles: Optical properties and implementations in cancer diagnosis and photothermal therapy, J. Adv. Res. 1 (2010) 13-28. doi:10.1016/j.jare.2010.02.002.

[17] R. Weissleder, A clearer vision for in vivo imaging., Nat. Biotechnol. 19 (2001) 316-317. doi:10.1038/86684.

[18] J.W. Nichols, Y.H. Bae, Odyssey of a cancer nanoparticle: from injection site to site of action, Nano Today. 7 (2012) 606-618. doi:10.1016/j.nantod.2012.10.010. Odyssey.

[19] S. Schrittwieser, B. Pelaz, W.J. Parak, S. Lentijo-Mozo, K. Soulantica, J. Dieckhoff, F. Ludwig, T. Altantzis, S. Bals, J. Schotter, Homogeneous Protein Analysis by Magnetic Core-Shell Nanorod Probes, ACS Appl. Mater. Interfaces. 8 (2016) 8893-8899. doi:10.1021/acsami.5b11925.

[20] E.C. Ximendes, W.Q. Santos, U. Rocha, U.K. Kagola, F. Sanz-Rodríguez, N. Fernández, A.D.S. Gouveia-Neto, D. Bravo, A.M. Domingo, B. Del Rosal, C.D.S. Brites, L.D. Carlos, D. Jaque, C. Jacinto, Unveiling in Vivo Subcutaneous Thermal Dynamics by Infrared Luminescent Nanothermometers, Nano Lett. 16 (2016) 1695-1703. doi:10.1021/acs.nanolett.5b04611.

[21] G. Armelles, A. Cebollada, A. García-Martín, J.M. García-Martín, M.U. González, J.B. González-Díaz, E. Ferreiro-Vila, J.F. Torrado, Magnetoplasmonic nanostructures: systems supporting both plasmonic and magnetic properties, J. Opt. A Pure Appl. Opt. 11 (2009) 114023. doi:10.1088/1464-4258/11/11/114023.

[22] M. Donolato, P. Antunes, R.S. Bejhed, T. Zardán Gómez De La Torre, F.W. Østerberg, M. Strömberg, M. Nilsson, M. Strømme, P. Svedlindh, M.F. Hansen, P. Vavassori, Novel readout method for molecular diagnostic assays based on optical measurements of magnetic nanobead dynamics, Anal. Chem. 87 (2015) 1622-1629. 
doi:10.1021/ac503191v.

[23] C. Peters, O. Ergeneman, P.D.W. García, M. Müller, S. Pané, B.J. Nelson, C. Hierold, Superparamagnetic twist-type actuators with shape-independent magnetic properties and surface functionalization for advanced biomedical applications, Adv. Funct. Mater. 24 (2014) 5269-5276. doi:10.1002/adfm.201400596.

[24] X.Z. Chen, M. Hoop, N. Shamsudhin, T. Huang, B. Özkale, Q. Li, E. Siringil, F. Mushtaq, L. Di Tizio, B.J. Nelson, S. Pané, Hybrid Magnetoelectric Nanowires for Nanorobotic Applications: Fabrication, Magnetoelectric Coupling, and Magnetically Assisted In Vitro Targeted Drug Delivery, Adv. Mater. 29 (2017). doi:10.1002/adma.201605458.

[25] J.B. González-Díaz, A. García-Martín, J.M. García-Martín, A. Cebollada, G. Armelies, B. Sepúlveda, Y. Alaverdyan, M. Käll, Plasmonic Au/Co/Au nanosandwiches with enhanced magneto-optical activity, Small. 4 (2008) 202-205. doi:10.1002/smll.200700594.

[26] B. Sepúlveda, J.B. González-Díaz, A. García-Martín, L.M. Lechuga, G. Armelles, Plasmon-induced magneto-optical activity in nanosized gold disks, Phys. Rev. Lett. 104 (2010) 147401. doi:10.1103/PhysRevLett.104.147401.

[27] G. Armelles, A. Cebollada, A. García-Martín, M.U. González, Magnetoplasmonics: Magnetoplasmonics: Combining Magnetic and Plasmonic Functionalities, Adv. Opt. Mater. 1 (2013) 10-35. doi:10.1002/adom.201370002.

[28] A. Espinosa, M. Bugnet, G. Radtke, S. Neveu, G.A. Botton, ClaireWilhelm, A. AbouHassan, Can magneto-plasmonic nanohybrids efficiently combine photothermia with magnetic hyperthermia?, Nanoscale. 7 (2015) 18872-18877. doi:10.1039/c5nr06168g.

[29] M. Abdulla-Al-Mamun, Y. Kusumoto, T. Zannat, Y. Horie, H. Manaka, Au-ultrathin functionalized core-shell (Fe3O4@ Au) monodispersed nanocubes for a combination of magnetic/plasmonic photothermal cancer cell killing, RSC Adv. 3 (2013) 7816-7827. doi:10.1039/c3ra21479f.

[30] I. Urries, C. Muñoz, L. Gomez, C. Marquina, V. Sebastian, M. Arruebo, J. Santamaria, Magneto-plasmonic nanoparticles as theranostic platforms for magnetic resonance imaging, drug delivery and NIR hyperthermia applications., Nanoscale. 6 (2014) 92309240. doi:10.1039/c4nr01588f.

[31] Z. Xu, Y. Hou, S. Sun, Magnetic Core/Shell Fe 3 O 4 /Au and Fe 3 O 4 /Au/Ag Nanoparticles with Tunable Plasmonic Properties, J. Am. Chem. Soc. 129 (2007) 86988699. doi:10.1021/ja073057v.

[32] W. Dong, Y. Li, D. Niu, Z. Ma, J. Gu, Y. Chen, W. Zhao, X. Liu, C. Liu, J. Shi, Facile Synthesis of Monodisperse Superparamagnetic Fe3O4 Core@hybrid@Au Shell Nanocomposite for Bimodal Imaging and Photothermal Therapy, Adv. Mater. 23 (2011) 5392-5397. doi:10.1002/adma.201103521.

[33] E. Fantechi, A.G. Roca, B. Sepúlveda, P. Torruella, S. Estradé, F. Peiró, E. Coy, S. Jurga, N.G. Bastús, J. Nogués, V. Puntes, Seeded Growth Synthesis of $\mathrm{Au}-\mathrm{Fe}_{3} \mathrm{O}_{4}$ Heterostructured Nanocrystals: Rational Design and Mechanistic Insights, Chem. Mater. 29 (2017) 4022-4035. doi:10.1021/acs.chemmater.7b00608.

[34] P. Hanarp, D.S. Sutherland, J. Gold, B. Kasemo, Control of nanoparticle film structure for colloidal lithography, Colloid Surf. A. 214 (2003) 23-36. doi:10.1016/S09277757(02)00367-9.

[35] J.I. Martín, J. Nogués, K. Liu, J.L. Vicent, I.K. Schuller, Ordered magnetic nanostructures: Fabrication and properties, J. Magn. Magn. Mater. 256 (2003) 449-501. doi:10.1016/S0304-8853(02)00898-3. 
[36] S.H. Chung, R.D. McMichael, D.T. Pierce, J. Unguris, Phase diagram of magnetic nanodisks measured by scanning electron microscopy with polarization analysis, Phys. Rev. B - Condens. Matter Mater. Phys. 81 (2010) 024410. doi:10.1103/PhysRevB.81.024410.

[37] D.K. Roper, W. Ahn, M. Hoepfner, Microscale heat transfer transduced by surface plasmon resonant gold nanoparticles, J. Phys. Chem. C. (2007) 3636-3641. doi:10.1021/jp064341w.

[38] L. Uba, A. Polewko, S. Uba, R. Gontarz, A.N. Yaresko, V.N. Antonov, Determination of the interfacial magneto-optical properties in sputtered Fe/Au multilayer structures, Phys. Stat. Sol. A. 196 (2003) 145-148. doi:10.1002/pssa.200306428.

[39] D. Regatos, D. Fariña, A. Calle, A. Cebollada, B. Sepúlveda, G. Armelles, L.M. Lechuga, $\mathrm{Au} / \mathrm{Fe} / \mathrm{Au}$ multilayer transducers for magneto-optic surface plasmon resonance sensing, $\mathrm{J}$. Appl. Phys. 108 (2010) 054502. doi:10.1063/1.3475711.

[40] M. Rohrer, H. Bauer, J. Mintorovitch, M. Requardt, H.J. Weinmann, Comparison of magnetic properties of MRI contrast media solutions at different magnetic field strengths, Invest. Radiol. 40 (2005) 715-724. doi:Doi 10.1097/01.Rli.0000184756.66360.D3.

[41] S. Huo, S. Jin, X. Ma, X. Xue, K. Yang, A. Kumar, P.C. Wang, J. Zhang, Z. Hu, X.J. Liang, Ultrasmall gold nanoparticles as carriers for nucleus-based gene therapy due to size-dependent nuclear entry, ACS Nano. 8 (2014) 5852-5862. doi:10.1021/nn5008572.

[42] J. Beik, Z. Abed, F.S. Ghoreishi, S. Hosseini-Nami, S. Mehrzadi, A. Shakeri-Zadeh, S.K. Kamrava, Nanotechnology in hyperthermia cancer therapy: From fundamental principles to advanced applications, J. Control. Release. 235 (2016) 205-221.

doi:10.1016/j.jconrel.2016.05.062.

[43] I.M.M. Paino, V.S. Marangoni, R. de C.S. de Oliveira, L.M.G. Antunes, V. Zucolotto, Cyto and genotoxicity of gold nanoparticles in human hepatocellular carcinoma and peripheral blood mononuclear cells, Toxicol. Lett. 215 (2012) 119-125. doi:10.1016/j.toxlet.2012.09.025.

[44] O. Penon, T. Patiño, L. Barrios, C. Nogués, D.B. Amabilino, K. Wurst, L. Pérez-García, A new porphyrin for the preparation of functionalized water-soluble gold nanoparticles with low intrinsic toxicity, ChemistryOpen. 4 (2015) 127-136. doi:10.1002/open.201402092.

[45] A. Espinosa, R. Di Corato, J. Kolosnjaj-Tabi, P. Flaud, T. Pellegrino, C. Wilhelm, Duality of Iron Oxide Nanoparticles in Cancer Therapy: Amplification of Heating Efficiency by Magnetic Hyperthermia and Photothermal Bimodal Treatment, ACS Nano. 10 (2016) 2436-2446. doi:10.1021/acsnano.5b07249.

[46] Z. Li, A. Lopez-Ortega, A. Aranda-Ramos, J.L. Tajada, J. Sort, C. Nogues, P. Vavassori, J. Nogues, B. Sepulveda, Simultaneous Local Heating/Thermometry Based on Plasmonic Magnetochromic Nanoheaters, Small. 14 (2018) 1800868. doi:10.1002/smll.201800868. 